\title{
Percepção dos pais sobre técnicas de controle comportamental na Clínica Odontopediátrica da Faculdade UniRuy, Salvador-BA
}

\author{
Parents' perception of behavioral control \\ techniques at the Pediatric Dentistry Clinic at \\ UniRuy Faculty, Salvador-BA
}

\section{Gabriela Xavante de Oliveira Santana Brito ${ }^{1}$ (1) Cíntia de Vasconcellos Machado 2 (1)}

\author{
${ }^{1}$ Centro Universitário UniRuy (Salvador). Bahia, Brasil. dragabrielaxavante@outlook.com \\ ${ }^{2}$ Autora para correspondência. Escola Bahiana de Medicia e Saúde Pública (Salvador). Bahia, Brasil. cintiamachado@bahiana.edu.br
}

\begin{abstract}
RESUMO | OBJETIVO: Avaliar a percepção dos pais e/ou responsáveis das crianças atendidas na clínica odontopediátrica da Faculdade UniRuy, sobre as técnicas de manejo comportamental que podem ser utilizadas durante $o$ atendimento infantil. METODOLOGIA: Para a realização da pesquisa, os pais e/ou responsáveis responderam a um questionário, onde havia uma breve explicação sobre determinadas técnicas de manejo do comportamento infantil, assim como se concordavam ou não com o uso das mesmas. Estavam presentes nos questionários as seguintes técnicas: falar-mostrar-fazer, comunicação não verbal, controle de voz, reforço positivo, distração, modelagem ou imitação, estabilização protetora e mão-sobre-a-boca. Todos os participantes do estudo responderam ao Termo de Consentimento Livre e Esclarecido. RESULTADOS: Foram respondidos 53 questionários, onde a técnica de controle do comportamento com maior aceitação pelos pais foi a de "falar-mostrar-fazer" (100\%) e a com maior rejeição foi a "mão-sobre-a-boca" (75\%), evidenciando uma maior rejeição por técnicas que restringem os movimentos da criança. CONCLUSÕES: As técnicas de controle comportamental visam reduzir a ansiedade e o medo da criança no ambiente odontológico, modulando o seu comportamento, afim de permitirem um atendimento seguro para o paciente, tanto do ponto de vista físico como emocional. Toda a equipe profissional deve ter o conhecimento e domínio de tais técnicas, assim como uma compreensão sobre os aspectos psicológicos das crianças em cada etapa do seu desenvolvimento. Da mesma forma, é crucial o entendimento e a permissão dos pais para a realização de técnicas que limitem os movimentos das crianças, como a estabilização protetora.
\end{abstract}

PALAVRAS-CHAVE: Odontopediatria. Controle comportamental. Crianças.

\begin{abstract}
OBJECTIVE: To evaluate the perception of parents and/or legal guardians of the children attended in the pediatric dentistry clinic at UniRuy Faculty about the behavioral management techniques that can be used during childcare. METHODOLOGY: In order to carry out the research, parents and/or guardians answered a questionnaire, where there was a brief explanation about certain techniques for managing child behavior, as well as whether or not they agreed with their use. The following techniques were present in the questionnaires: tell-show-do, non-verbal communication, voice control, positive reinforcement, distraction, modeling or imitation, protective stabilization, and hand-over-mouth. In addition, all study participants responded to the Free and Informed Consent Form. RESULTS: 53 questionnaires were answered, and the results showed that the behavior control technique with the greatest acceptance by parents was "tell-show-do" (100\%), and the one with the greatest rejection was "hand-over-mouth" (75\%), showing greater rejection by techniques that restrict the child's movements. CONCLUSIONS: The behavioral control techniques aim to reduce the child's anxiety and fear in the dental environment, modulating their behavior to allow safe care for the patient, both physically and emotionally. The entire professional team must have the knowledge and domain of such techniques and an understanding of the psychological aspects of children at each stage of their development. Likewise, parent's understanding and permission to perform techniques that limit children's movements, such as protective stabilization, is crucial.
\end{abstract}

KEYWORDS: Pediatric Dentistry; Behavior control; Child. 


\section{Introdução}

Na clínica odontopediátrica, é comum que o profissional se depare com determinados comportamentos como a ansiedade, o medo, assim como a apreensão das crianças e também dos pais em relação ao tratamento odontológico. Para que a consulta odontológica ocorra da melhor forma possível, o cirurgião dentista precisa estar apto a realizar o atendimento de uma maneira cautelosa e explicativa. É necessário conhecer a história do paciente como um todo, tanto pelo lado social, como familiar, entender as experiências passadas, e, através das informações obtidas pelos pais, saber como conduzir a criança no atendimento, demonstrando confiança para a relação profissional-paciente..$^{1,2}$

Diversos são os fatores que podem influenciar o comportamento da criança na clínica odontológica, tais como a idade, maturidade, relacionamento com os pais, presença de dor, abordagem da equipe profissional, assim como as experiências prévias do paciente. ${ }^{3}$ Em Odontopediatria, o sucesso das consultas de prevenção e dos procedimentos curativos está relacionado com a gestão comportamental realizada durante o tratamento ${ }^{2}$, e isso depende do relacionamento e interação entre pais, cirurgião-dentista, paciente, e de toda a equipe de trabalho da clínica. ${ }^{4}$

É de fundamental importância conhecer e identificar os diversos tipos de comportamentos que as crianças podem apresentar no ambiente odontológico, além do conhecimento mínimo de psicologia considerado normal para cada faixa etária. ${ }^{3}$ Com o objetivo de reduzir o medo, a ansiedade e também a birra do paciente odontopediátrico, técnicas farmacológicas e não farmacológicas podem ser empregadas, a fim de estabelecer uma atitude positiva da criança e também dos responsáveis, permitindo um atendimento odontológico mais seguro, física e psicologicamente. ${ }^{3}$

As técnicas de controle ou manejo comportamental na clínica odontopediátrica destritas na literatura incluem: falar-mostrar-fazer, comunicação não verbal, controle de voz, reforço positivo, distração, modelagem ou imitação, estabilização protetora e mão-sobrea-boca. ${ }^{3-5} \mathrm{~A}$ seguir, as referidas técnicas são descritas.

- Falar-mostrar-fazer: é a técnica mais utilizada na odontopediatria e consiste em uma explicação verbal sobre os procedimentos que serão realizados, utilizando-se para isso uma linguagem adequada a cada faixa etária e nível de compreensão da criança. Em seguida, deve-se realizar uma demonstração dos materiais, instrumentos e aparelhos que serão utilizados, e após realizar o procedimento propriamente dito. Essa técnica pode diminuir bastante a ansiedade da criança e o medo dos ruídos, por exemplo, algo comum no consultório odontológico, e que podem causar choro e inquietação. ${ }^{-}$Essa técnica é amplamente aceita e efetiva, embora menos sucesso seja observado em crianças muito pequenas.

- Comunicação não verbal: consiste no contato com a criança, sem que haja comunicação verbal. Pode ocorrer através de um abraço, um toque, um carinho, a maneira de olhar, de sorrir. Receber a criança com um sorriso ou um abraço carinhoso na clínica já pode eliminar ou diminuir a tensão ou apreensão da criança quando chega ao ambiente odontológico. Tem como objetivos melhorar a efetividade de outras técnicas de abordagem baseadas na comunicação verbal, além de obter e manter a atenção do paciente e do acompanhante.

- Controle de voz: nesta situação, há uma mudança controlada na tonalidade da voz e expressão facial do cirurgião-dentista, para influenciar e direcionar o comportamento do paciente, chamando sua atenção, com o objetivo de conduzi-lo a um estado de tranquilidade de conforto.

- Reforço Positivo: nesta técnica, há uma gratificação da criança pelo comportamento positivo apresentado durante o atendimento, para que ela fixe e repita este comportamento adequado. $O$ reforço positivo pode ser social, através de elogios, expressão facial alegre, demonstrações de afeto, ou não social, como a utilização de lembrancinhas ou "prêmios" ao final da consulta.

- Distração: É usada para desviar a atenção da criança do procedimento em si, utilizando-se para isso músicas, vídeos, brinquedos, histórias, desenhos ou bonecos.

- Modelagem ou imitação: Nesta técnica, é realizado o procedimento em outro paciente (normalmente mais velho e com comportamento adequado) ou bonecos, servindo de modelo, encorajando a criança antes do seu atendimento, mostrando a ela que aquela situação não oferece "riscos" para ela. 
- Estabilização protetora: É uma técnica que restringe os movimentos físicos da criança durante o atendimento, minimizando os riscos de injúrias e danos durante $o$ atendimento odontopediátrico, proporcionando um atendimento mais seguro para a criança, pais e equipe. Os pais ou responsáveis devem autorizar o uso da estabilização protetora e, na maior parte das vezes, auxiliam o dentista e sua equipe na realização desta. É utilizada principalmente em crianças na primeira infância, pela maior imaturidade e capacidade de compreensão. É imprescindível que haja a autorização dos pais ou responsáveis para a realização desta técnica. Pode ser realizada de forma ativa, onde os pais ou alguém da equipe seguram fisicamente a criança ou por meio de dispositivos conhecidos como pedi-wrap, que enrolam a criança em um tecido próprio para este fim, impedindo seus movimentos.

- Mão-sobre-a-boca: nesta técnica, o dentista coloca a mão delicadamente sobre a boca da criança (para que ela não consiga gritar) e fala no ouvido da mesma, para que ela consiga ouvir o profissional. Normalmente a criança está gritando muito e normalmente o choro é sem lágrimas. Esta técnica é bastante controversa e totalmente contraindicada para pacientes muito pequenos ou pacientes incapazes de compreender cognitivamente as explicações do dentista.

De acordo com alguns autores, a aceitação e percepção dos pais sobre técnicas de manejo comportamental não farmacológico é um ponto de extrema importância. Muitos pais não aceitam que sejam utilizadas determinadas técnicas de controle do comportamento infantil, em especial, a estabilização protetora, pois acreditam que o cirurgião-dentista contendo o seu filho pode levar a um "trauma psicológico" ou até por medo da reação da criança, muitas vezes, por falta da explicação e entendimento de tais técnicas. Desde que a estabilização seja feita no momento exato e da forma correta, dificilmente vai acarretar em algum trauma psicológico para a criança. Após a criança cooperar no procedimento, imediatamente é feito o reforço positivo, elogiando e reforçando o comportamento positivo apresentado. ? $^{-}$

É fundamental que as técnicas de manejo do comportamento sejam explicadas aos pais, com a intensão de deixá-los seguros quanto à realização das mesmas. Os pais precisam estar cientes que as técnicas de manejo comportamental utilizadas são empregadas sempre com o objetivo de proporcionar um meIhor atendimento e bem estar aos pacientes infantis.
Desta forma, o objetivo deste trabalho foi avaliar a percepção dos pais e/ou responsáveis das crianças atendidas na clínica odontopediátrica da Faculdade UniRuy sobre as técnicas de manejo comportamental que podem ser utilizadas durante $o$ atendimento infantil.

\section{Material e métodos}

O presente estudo consistiu de uma avaliação transversal descritiva, onde a percepção dos pais e/ou responsáveis acerca das técnicas de controle do comportamento infantil na clínica odontopediátrica foi avaliada através de um questionário.

Fizeram parte da pesquisa os pais ou responsáveis de crianças entre 5 e 12 anos de idade que estavam em atendimento nas disciplinas Materno Infantil, Clínica Odontopediátrica e Cariologia da Faculdade UniRuy, Salvador-BA, nos meses de março a abril de 2019. Os dados foram coletados na sala de espera, enquanto os pais ou responsáveis estavam aguardando o atendimento das crianças. Os pais que concordaram em participar da pesquisa assinaram um termo de consentimento livre e esclarecido.

No questionário, os pais deveriam responder se concordavam ou não com o uso das seguintes técnicas de controle do comportamento durante o atendimento odontológico dos seus filhos: falar-mostrar-fazer, comunicação não verbal, controle da voz, reforço positivo, distração, modelagem ou imitação, estabilização protetora e técnica da mão-sobre-a-boca. Não foram dadas explicações verbais para os pais no momento em que respondiam ao questionário. Entretanto, o questionário continha uma breve descrição de cada uma das técnicas de modulação do comportamento infantil, numa linguagem acessível ao leigo. Foi respeitada a confidencialidade e anonimato dos sujeitos da pesquisa. A pesquisa não acarretou qualquer risco nem custo para os participantes. O questionário aplicado para os pais e/ou responsáveis foi elaborado pelo pesquisador.

Após a coleta dos dados, os mesmos foram tabulados em uma planilha no Microsoft Excel e foi realizada uma análise descritiva para observar qual a técnica possuía maior índice de aceitação e qual possuía o maior índice de rejeição pelos pais e/ou responsáveis. Os critérios de inclusão foram pais ou responsáveis de crianças que apenas eram atendidas na clínica odontopediátrica da Faculdade UniRuy. 
Este trabalho foi submetido ao Comitê de Ética e Pesquisa do Hospital da Bahia, Salvador-BA, tendo sido aprovado sob o número CAAE 13084219.1.0000.5606.

\section{Resultados}

Foram entregues termos de consentimento e questionários sobre as técnicas de controle comportamental a 53 pais e/ou responsáveis de crianças que estavam sendo atendidas na clínica odontológica da Faculdade UniRuy, onde os mesmos responderam SIM para as técnicas em que aceitariam que fossem utilizadas em seus filhos e NÃO para as técnicas que não aceitariam que fossem utilizadas. As crianças que estavam sendo atendidas na clínica odontopediátrica possuíam idades entre 5 e 12 anos. A técnica que obteve a maior aceitação pelos pais foi a de falar-mostrar-fazer (100\%), seguida da técnica de distração (98\%). A técnica que obteve o maior índice de rejeição foi a de mão-sobre-a-boca (75\%), seguida da técnica da contenção física $(18,86 \%)$. Os resultados estão descritos no Quadro 1.

Quadro 1. Aceitação dos pais e/ou responsáveis de crianças atendidas na clínica odontopediátrica da Faculdade UniRuy, Salvador-BA, em relação às técnicas de controle do comportamento infantil que podem ser utilizadas durante o atendimento clínico

\begin{tabular}{|c|c|c|}
\hline \multirow{2}{*}{$\begin{array}{c}\text { TÉCNICAS } \\
\text { DE CONTROLE DO } \\
\text { COMPORTAMENTO INFANTIL }\end{array}$} & \multicolumn{2}{|c|}{ ACEITAÇÃO DOS PAIS E/OU RESPONSÁVEIS } \\
\hline & SIM & NÃO \\
\hline Falar-mostrar-fazer & 53 & 0 \\
\hline Comunicação não verbal & 45 & 8 \\
\hline Controle por voz & 48 & 5 \\
\hline Reforço positivo & 50 & 3 \\
\hline Distração & 51 & 2 \\
\hline Modelagem ou imitação & 46 & 7 \\
\hline Estabilização protetora & 43 & 10 \\
\hline Mão-sobre-a-boca & 28 & 25 \\
\hline
\end{tabular}

\section{Discussão}

O atendimento odontológico infantil requer o gerenciamento do comportamento da criança de forma a possibilitar o exame e intervenções objetivando a promoção da saúde. Desta forma, a abordagem da criança ocorre através da utilização de técnicas de controle do comportamento adequadas à idade, nível socioeconômico, estado de saúde geral e bucal da criança, assim como fatores familiares. Diante do exposto, torna-se relevante o conhecimento e domínio pelo cirurgião-dentista das técnicas de controle do comportamento do paciente infantil com o intuito de auxiliar o clínico geral e o Odontopediatra na abordagem da criança durante o atendimento clínico. $\frac{8}{}$ 
O critério fundamental para aplicação das técnicas de controle do comportamento infantil é a obtenção da permissão dos pais ou responsáveis pela criança através do termo de consentimento. Porém, segundo a literatura, a maioria dos profissionais que faz uso dessas técnicas não obtém o consentimento prévio dos pais por escrito. ${ }^{?}$

O resultado do estudo realizado por Fúccio e colaboradores $^{9}$ revela que o mais importante durante o atendimento infantil é uma relação harmônica e cercada de confiança entre o profissional, os pais e o paciente, o que também é evidenciado por outros autores. ${ }^{10}$ Quando os pais estão certos de que o Odontopediatra está preocupado com a saúde e o bem estar da criança, são, na maioria das vezes, fortes aliados para o adequado andamento do tratamento. No entanto, torna-se necessário registrar que algumas técnicas exigem que os pais as aprovem e assinem o termo de consentimento específico, como a técnica da estabilização protetora, a qual restringe os movimentos da criança.

Perante um comportamento cooperativo ou não cooperativo da criança, pode-se aplicar a técnica que melhor se adeque a cada situação clínica e a cada paciente, desde que esta vise a segurança e o conforto do paciente durante o atendimento. 5 Segundo Possobon e colaboradores 11 , a criança está sujeita à influência de comportamento e postura dos pais, sendo também influenciada pela equipe do consultório odontológico, porém, em menor magnitude.

Meira Filho et al. $\frac{12}{}$ realizaram uma pesquisa com as mães na sala de espera, enquanto os seus filhos estavam sendo atendidos no Programa de Saúde da Família Vila Lacasa, na cidade de Cachoeirinha-PE. Os autores observaram que no momento em que foram questionadas a respeito de sua percepção sobre a vivência odontológica de seus filhos, se o comportamento dos filhos era positivo ou não durante a realização do procedimento odontológico, $60 \%$ das mães consideraram-na positiva e $94 \%$ relataram que o comportamento do cirurgião-dentista teve influência nesse aspecto, em relação a ter um maior acolhimento, paciência e dedicação com a criança durante $o$ atendimento odontológico, evidenciando a importância do preparo, experiência e conhecimento da equipe odontológica na condução da consulta odontológica infantil.
Dentre as técnicas utilizadas na odontopediatria, a falar-mostrar-fazer foi a mais bem aceita pelos pais no presente estudo, apresentando um percentual de $100 \%$ de aceitação, seguida da distração (98\%). A que obteve maior índice de rejeição foi a técnica da mão-sobre-a-boca (75\%), seguida da contenção física (18, 86\%). No estudo de Acharya ${ }^{13}$, a técnica de manejo comportamental de maior aceitação pelos pais foi igualmente a de falar-mostrar-fazer (86\%), seguida do reforço positivo (76\%). A técnica menos aceita no referido estudo foi a técnica da mão-sobre-a-boca, apresentando, desta maneira, resultados semelhantes ao presente estudo.

Fúccio et al. ${ }^{9}$ realizaram um estudo com 49 pais que permaneciam na sala de espera enquanto o filho recebia atendimento na Clínica de Odontopediatria do Departamento de Odontopediatria e Ortodontia da Faculdade de Odontologia da Universidade Federal de Minas Gerais. Os pais foram conduzidos a uma sala e foi feita uma exposição audiovisual correspondente às seguintes técnicas: falar-mostrar-fazer, controle da voz, reforço positivo, modelagem ou imitação, mão-sobre-a-boca, estabilização proteto$r a$, sedação e anestesia geral. Os pais precisavam marcar em um diagrama o grau de aceitação dessas técnicas. Como resultado desse estudo, observou-se que as técnicas consideradas não-restritivas (falarmostrar-fazer, controle da voz, reforço positivo e modelagem ou imitação) foram aceitas, em média, por $81 \%$ dos pais; $15 \%$ às vezes autorizariam a utilização de tais técnicas durante o tratamento odontológico do filho, não tinham certeza se aceitariam ou não e $4 \%$ nunca aceitariam. A técnica falar-mostrarfazer foi a mais aceita pelos pais (98\%), seguida pelo reforço positivo (91,8\%). Das técnicas não-restritivas, as menos aceitas pelos pais foram controle da voz $(8,2 \%)$ e modelagem ou imitação $(6,1 \%)$, concordando, mais uma vez, com os resultados do presente estudo, onde houve uma maior rejeição dos pais com relação as técnicas mão-sobre-a-boca e contenção física. A rejeição dos pais com relação à técnica da modelagem ou imitação pode ter como justificativa o fato de o profissional não explicar como funciona a técnica de uma forma em que seja bem entendido, quais os objetivos e benefícios que a mesma pode trazer para a criança, como forma de benefício, por exemplo, pode-se citar o encorajamento. 
Em 2009, Machado e colaboradores ${ }^{7}$ realizaram um estudo no qual foi possível observar que a aceitação dos responsáveis foi positiva quando existia uma explicação prévia acerca das técnicas de manejo comportamental que seriam realizadas no momento do atendimento.

Com relação à indicação da técnica da mão-sobre-aboca, o erro mais frequente ocorre quando o cirurgião-dentista aplica esse procedimento em pacientes que não estão aptos a recebê-lo, ou seja, em crianças com idade menor do que três anos, com algum tipo de deficiência mental, medrosas, tímidas e ansiosas. Estas falhas ocorrem, normalmente, devido à falta de conhecimento específico sobre 0 assunto. ${ }^{14}$ Embora a American Academy of Pediatric Dentistry ${ }^{3}$ reconheça as indicações e contraindicações para o uso dessa técnica, atualmente, ela é pouco utilizada no âmbito odontológico. Inclusive, de acordo com a Associação Brasileira de Odontopediatria ${ }^{4}$, a técnica da mão-sobre-a-boca e suas variações não são recomendadas em hipótese alguma.

O profissional precisa se comunicar de forma correta com a criança e seus pais e/ou responsáveis para que a visita ao consultório seja menos traumática possível. O principal objetivo da comunicação é a compreensão das crianças. É importante oferecer garantias de que a situação será menos ameaçadora possível, o que exige do profissional uma atuação assertiva e que transmita segurança e tranquilidade para que a permanência na cadeira do dentista ocorra sem maiores transtornos. ${ }^{1}$ Apesar de alguns pais não aprovarem na pesquisa a técnica da estabilização protetora, é uma das técnicas mais utilizadas na odontopediatria, quando são bem explicadas e deixando tudo esclarecido para os pais, é bem sucedi$\mathrm{da}$, uma vez que os mesmos aceitam que é a melhor maneira de oferecer segurança à criança durante o atendimento odontológico. É importante que a criança esteja numa posição o mais confortável possível, sendo realizada junto a todas as outras técnicas não restritivas de manejo do comportamento, como distração, reforço positivo, entre outras. ${ }^{4}$

Somando-se o conhecimento e emprego adequado das técnicas de manejo do comportamento infantil, sempre que possível, a primeira consulta odontológica da criança deve ser de prevenção e não em uma situação de urgência, principalmente com a presença de dor, o que dificulta um comportamento positivo nesta situação, ressaltando a importância da educação em saúde e das consultas de prevenção e manutenção da saúde bucal das crianças., 45,16 Da mesma forma, o profissional deve otimizar o seu tempo de trabalho com planejamento e organização, pois a criança, principalmente as pequenas, não toleram tempos muito longos de consulta. ${ }^{4}$ Os pais devem estar sempre presentes no consultório quando crianças menores de três anos estão sendo atendidas, por se sentirem mais seguras na presença dos pais. ${ }^{4}$ Para as crianças maiores, pode haver uma negociação da presença ou não dos pais na sala de atendimento no caso de birra, embora essa conduta não tenha evidências científicas na literatura da sua efetividade. ${ }^{4,17}$

Por fim, é necessário que o profissional conheça e respeite cada fase do desenvolvimento da criança, sua capacidade de compreensão, seus medos e anseios, assim como os anseios dos pais ou responsáveis, para que as técnicas de controle do comportamento possam ser empregadas de forma efetiva e de forma adequada para os diversos níveis de entendimento e maturidade apresentados pelas crianças. 2.18

\section{Conclusões}

Os resultados deste estudo permitiram concluir que as técnicas de controle do comportamento infantil que possuíram uma maior aceitação dos pais e/ou responsáveis foram falar-mostrar-fazer, seguida da técnica da distração. As técnicas que possuíram uma maior rejeição foram mão-sobre-a-boca, seguida da contenção física, mostrando uma maior dificuldade de aceitação dos pais por técnicas que restringem os movimentos da criança.

\section{Contribuições dos autores}

Brito GXOS participou da concepção, delineamento, coleta dos dados, interpretação dos resultados e redação do artigo científico. Machado CV participou da concepção, delineamento, interpretação dos resultados, redação e orientação do artigo científico. 


\section{Conflitos de interesses}

Nenhum conflito financeiro, legal ou político envolvendo terceiros (governo, empresas e fundações privadas, etc.) foi declarado para nenhum aspecto do trabalho submetido (incluindo, mas não se limitando a subvenções e financiamentos, participação em conselho consultivo, desenho de estudo, preparação de manuscrito, análise estatística, etc.).

\section{Referências}

1. Albuquerque CM, Gouvêa CVD, Moraes RCM, Barros RN, Couto CF. Principais técnicas de controle de comportamento em Odontopediatria. Arq Odontol [Internet]. 2010;45(2):110-5. Disponível em: http://revodonto.bvsalud.org/pdf/aodo/v46n2/ a08v46n2.pdf

2. Sato CM, Abanto J, Bönecker M, Corrêa MSNP. Gestão comportamental em odontopediatria baseada em evidências científicas. In: Bonecker M, Abanto J, Imparato JCP, Correa MSNP, Guedes-Pinto AC. Odontopediatria: Evidências científicas para a conduta clínica em bebês e pré-escolares. São Paulo: Quintessence; 2018. p.201-9.

3. American Academy of Pediatric Dentistry. Behavior guidance for the pediatric dental patient [Internet]. Chicago, III.: American Academy of Pediatric Dentistry; 2020. Diposnível em: https:// www.aapd.org/globalassets/media/policies guidelines/bp behavguide.pdf

4. Associação Brasileira de Odontopediatria. Diretrizes para procedimentos clínicos em Odontopediatria. Rio de Janeiro: Santos; 2021. p.27-36.

5. Silva LFP, Freire NC, Santana RS, Miasato JM. Técnicas de manejo comportamental não farmacológicas na odontopediatria. Rev Odontol Univ Cid São Paulo [Internet]. 2016;28(2):13542. https://arquivos.cruzeirodosuleducacional.edu.br/ principal/old/revista_odontologia/pdf/maio_agisto_2016/ Odonto 022016 135-142 1.pdf

6. Brandemburg OJ, Haydu VB. Contribuições da análise de comportamento em odontopediatria. Psicol cienc prof. 2009;29(3):462-75. https://doi.org/10.1590/S141498932009000300004

7. Machado MS, Nagano HCM, Silva JYB, Bosco VL. Participação dos pais na tomada de decisões no atendimento odontológico de seus filhos. Rev Odontol Univ Cid São Paulo [Internet]. 2009;21(1):38-47. Disponível em: https://publicacoes.unicid.edu. br/index.php/revistadaodontologia/article/view/434

8. Ferreira JMS, Aragão AKR, Colares V. Técnicas de controle do comportamento do paciente infantil: revisão de literatura. Pesq Bras Odontoped Clin Integr [Internet]. 2009;9(2):247-51. Disponível em: https://www.redalyc.org/pdf/637/63712851018.pdf
9. Fúccio F, Ferreira KD, Watanabe AS, Ramos-Jorge ML, Pordeus IA, Paiva SM. Aceitação dos pais em relação às técnicas de manejo do comportamento utilizadas em odontopediatria. J Bras Odontopediatr Odontol Bebê [Internet]. 2003;6(30):14651. Disponível em: https://www.dtscience.com/wp-content/ uploads/2015/11/Aceitação-dos-Pais-em-Relação-às-Técnicas-deManejo-do-Comportamento-Utilizadas-em-Odontopediatria.pdf

10. Zhou Y, Cameron E, Forbes G, Humphris G. Systematic review of the effect of dental staff behaviour on child dental patient anxiety and behaviour. Patient Educ Couns. 2011;85(1):4-13. https://doi.org/10.1016/j.pec.2010.08.002

11. Possobon RF, Moraes ABA, Costa Junior AL, Ambrosano GMB. O comportamento de crianças durante atendimento odontológico. Psic: Teor e Pesq. 2003;19(1):059-64. https://doi. org/10.1590/S0102-37722003000100008

12. Meira Filho MMO, Araújo DTC, Menezes VA, Garcia AFG. Atendimento odontológico da criança: percepção materna. RGO [Internet]. 2009;57(3):311-5. Disponível em https://www.researchgate.net/profile/Ana-Granville-Garcia/ publication/26872667_The_child's_dental_treatment_maternal_ perception/links/0c960536360addc939000000/The-childs-dentaltreatment-maternal-perception.pdf

13. Acharya S. Parental acceptance of various behaviour management techniques used in pediatric dentistry: a pilot study in Odisha, India. Pesq Bras Odontoped Clin Integr [Inernet]. 2017;17(1):1-6. Disponível em: http://revista.uepb.edu.br/index. php/pboci/article/view/3728

14. Bijella MFTB, Peres AS, Bijella MFB, Menezes JVNB, Vieira ALF, Matinhon CCR, et al. A utilização da técnica da Mão-Sobrea-Boca (MSB) em Odontopediatria - Revisão de literatura. J Bras Odontopediatr Odontol Bebê [Internet]. 2003;6(30):1639. Disponível em: https://www.dtscience.com/wp-content/ uploads/2015/11/A-Utilização-da-Técnica-da-Mão-Sobrea-BocaMSB-em-Odontopediatria---Revisão-de-Literatura.pdf

15. Singh KA, Moraes ABA, Bovi Ambrosano GM. Medo, ansiedade e controle relacionado ao tratamento odontológico. Pesq Odont Bras. 2000;14(2):131-6. https://doi.org/10.1590/S151774912000000200007

16. Meneses GR, Sakashita MS, Antonio RC, Rolim VCLB, CunhaCorreia AS. Comportamento da criança perante a presença das mães durante a assistência odontológica. Arch Health Invest. 2017;6(2):59-64. https://doi.org/10.21270/archi.v6i2.1782

17. De Luca MP, Massignan C, BolaN M, OliveirA LB, AydinoZ S, Dick $B$, et al. Does the presence of parents in the dental operatory room influence children's behaviour, anxiety and fear during their dental treatment? A systematic review. Int J Paediatr Dent. 2021;31(3):318-36. https://doi.org/10.1111/ipd.12762

18. Rezende MCRA, Lopes MRANE, Gonçalves DA, Zavanelli AC, Fajardo RS. Acolhimento e bem estar no atendimento odontológico humanizado: o papel da empatia. Arch Health Invest [Internet]. 2015;4(3):57-61. Disponível em: https://www. archhealthinvestigation.com.br/ArcHI/article/view/904 